\title{
How to Accelerate Very Early Recovery of Small Incision Lenticular Extraction Formyopia
}

Qinghong Lin ( $\sim 532884338 @ q q . c o m$ )

Bright Eye Hospital, Fuzhou 361000, China

Qing Li

Bright Eye Hospital, Fuzhou 361000, China

Zhengwei Shen

Bright Eye Hospital, Fuzhou 361000, China

\section{Research Article}

Keywords: Myopia, Small incision lenticule extraction, Fluorometholone, Very early phase

Posted Date: February 19th, 2021

DOI: https://doi.org/10.21203/rs.3.rs-199112/v1

License: (c) (i) This work is licensed under a Creative Commons Attribution 4.0 International License. Read Full License 
How to Accelerate Very Early Recovery of Small Incision Lenticular Extraction for Myopia

Qinghong Lin (MD) ${ }^{1}$, Qing Li (MD) ${ }^{1}$, Zhengwei Shen (MD) ${ }^{1 *}$

${ }^{1}$ Bright Eye Hospital, Fuzhou 361000, China

*Corresponding Author:

Zhengwei Shen, MD

Bright Eye Hospital, No.47 Wuyi Road, Fuzhou 361000, China.

Tel.: +8613606918326; E-mail: linqh19870624@163.com (6)

(7)

8

9

20



56

\begin{abstract}
Significance: A relatively long period of time is required for visual recovery after small incision lenticule extraction (SMILE), limiting its possible widespread application. The aim of this study was to compare the impact of different defined daily dose systems of $0.1 \%$ fluorometholone in the very early phase after SMILE operation.
\end{abstract}

Methods: One hundred and eighty patients (360 eyes) treated with SMILE were divided into three groups. Group A: 60 patients (120 eyes were not given any $0.1 \%$ FML; Group B: 60 patients (120 eyes, were given 0.1\% fluorometholone once every hour); Group C: 60 patients (120 eyes, were given 0.1\% fluorometholone every half an hour). Visual acuity, incidence of subject symptoms and optical quality were assessed at 2, 4 and $24 \mathrm{~h}$ postoperatively.

Results: The optical quality in group $\mathrm{C}$ was significantly higher than the other two groups $(\mathrm{p}<0.0001)$. The incidence of subject symptoms in group $\mathrm{C}$ was also notably lower. There were no significant differences between patient groups at $24 \mathrm{~h}$.

Conclusions: In conclusion, corneal healing, visual and optical quality assessment of patients treated with $0.1 \%$ fluorometholone $(\mathrm{q} 1 / 2 \mathrm{~h})$ in the first $2 \mathrm{~h}$ after SMILE were significantly improved in the very early postoperative phase.

Keywords: Myopia, Small incision lenticule extraction, Fluorometholone, Very early phase (1) 


\section{Introduction}

Small incision lenticule extraction is a new type of procedure for treating refractive errors, especially myopia and myopia astigmatism. It was developed on the basis offemtosecond laser-assisted in situ keratomileusis (LASIK) and is now considered a safer procedure, as it has a reduced risk of postoperative corneal flap complications ${ }^{1}$. Small incision lenticule extraction has been reported to have good long term outcomes in terms of safety, efficacy, predictability, and stability ${ }^{2,3}$. Nonetheless, compared to LASIK, it has a longer visual recovery time ${ }^{4,5}$ which limits its application. Currently very little is known about the influence of different treatment regimens on patient recovery during the very early phase post Small incision lenticule extraction, i.e. within the first two hours.

The use of topical $0.1 \%$ fluorometholone has shown a faster resolution of post-operative symptoms and reduction of the very early inflammatory and wound healing responses after Small incision lenticule extraction ${ }^{6}$.This study aimed to investigate the impact of $0.1 \%$ fluorometholone in different defined daily dose systems of $0.1 \%$ fluorometholone in the very early phase (within $2 \mathrm{~h}$ ) after Small incision lenticule extraction. Visual acuity and a series of visual quality-related indexes were evaluated at 2, 4 and $24 \mathrm{~h}$ postoperatively.

\section{Materials and Methods}

\section{Ethics statement}

All interventions approved by the Institutional Review Board of Fujian Medical University (Fuzhou, China), and were conducted according to the principles in the Declaration of Helsinki.

\section{Participants and procedures}

In the present study, 180 patients (360 eyes) who underwent Small incision lenticule extraction procedure at our center from July 2019 to November 2019 were recruited. Small incision lenticule extraction was performed using the Visu Max fem to second laser (Zeiss, Oberkochen, Germany). The following femtosecond laser parameters were used: $120 \mu \mathrm{m}$ cap thickness, $7.5 \mathrm{~mm}$ diameter of the cap, and $6.5 \mathrm{~mm}$ diameter of the posterior lenticule surface. A $2 \mathrm{~mm}$ long $\left(32^{\circ}\right)$ corneal incision was 
made at the 11 o'clock position. The remaining tissue bridges were disrupted using a thin, blunt spatula, and the lenticule was removed through the small incision with microforceps.

All subjects were divided into three groups depending on different defined daily dose systems of $0.1 \%$ fluorometholone administered during the first $2 \mathrm{~h}$ after Small incision lenticule extraction. Group A: 60 patients (120 eyes, control group) were not given any $0.1 \%$ fluorometholone , Group B: 60 patients (120 eyes) were given $0.1 \%$ fluorometholone (once every hour, qh); Group C: 60 patients (120 eyes) were given $0.1 \%$ fluorometholone (every half hour, q $1 / 2 \mathrm{~h}$ ).

\section{Measurement of visual indicators}

Optical Quality Analysis System II (OQAS, Visiometrics,Terrassa, Spain) was used to collect retinal images derived from an electric light source and analyze theirpoint spread function characteristics using thedual-channel technique ${ }^{7}$. The visual quality-related indexes, including cutoff for modulation transfer function, Strehl ratio and objective scattering index were produced and compared with visionor contrast sensitivity. These measures canreflectbetter the muddy degree of the refractive media of human eyesand define the source of visual quality problems objectively. SR indicates the ratio of the light intensity of theGaussian image point between defective and perfect refractive media ( 0.15 in healthy adults), in which a higher value represents better visual quality. The modulation transfer function cutoff is the value near which the spatial frequency represents the peak resolving capacity in the modulation transfer function curves. The modulation transfer function cutoff is $\geqslant 30$ cpd in adults with normal vision; and higher value represents better visual quality. Objective scattering index is $<2$ for healthy adults, and higher value represents muddier refractive media and worse visual quality. Modulation transfer function, Strehl ratio and objective scattering index were measured at $24 \mathrm{~h}$ preoperatively and 2 , 4 and 24 h postoperatively. Visual acuity (logMAR) and incidence of subjective symptoms were also assessed at 2, 4 and $24 \mathrm{~h}$ postoperatively.

Patients were asked for the presence of subjective symptoms, namely foreign body sensation, eye soreness, eye dryness and blurred vision. The incidence among the 
three groups was recorded at 2, 4 and $24 \mathrm{~h}$ after Small incision lenticule extraction.

\section{Statistical analysis}

All statistical analyses were performed using SPSS 18.0 (SPSS Inc., Chicago, Illinois, USA). Two-way ANOVA was applied to compare differences in visual acuity,incidence of subjective symptoms, objective scattering index, modulation transfer function cutoff, and Strehl ratio between these three groups. A p value of $<$ 0.05 was considered statistically significant.

\section{Results}

\section{Study subjects}

The demographics of the study population are summarizedin Table 1. No significant differences were found in terms of corrected distance visual acuity $(\log M A R)$, mean spherical equivalent, or intraocular pressure. The age and sex distributions were also comparable between the three groups. Notably there were no intra-operative complications.

\section{Impact of $0.1 \%$ fluorometholone treatment}

To further study the impact of different defined daily dose systems of $0.1 \%$ fluorometholone, we conducted visual tests for each group of patients (Fig.1). The visual acuity of all patients had recovered to below 0 at $24 \mathrm{~h}$ after operation, indicating that Small incision lenticule extraction is an effective procedure for myopia treatment. However, notably at 2 and $4 \mathrm{~h}$ after surgery, the visual acuity of group $\mathrm{C}$ was significantly better compared to the other two groups. Number of eyes recovering higher than $1.0(\log M A R \quad 0.0)$ were significantly higher in group C compared to Group A and B (114 eyes at $2 \mathrm{~h}$ and 119 eyes at $4 \mathrm{~h} 35$ eyes at $2 \mathrm{~h}$ and 86 eyes at $4 \mathrm{~h}$ vs 8 eyes at $2 \mathrm{~h}$ and 38 eyes at $4 \mathrm{~h}$ respectively). Significant differences in modulation transfer function cutoff, Strehl ratio and objective scattering index were also seen between different treatment groups at 2 and $4 \mathrm{~h}$ postoperatively, summarized in Tables 2 and 3. Group $\mathrm{C}$ had higher modulation transfer function cutoff, Strehl ratio and objective scattering index compared to the rest. No differences were detected in all examined parameters $24 \mathrm{~h}$ after surgery, summarized in Table 4.

We also investigated the incidence of subjective symptoms among the three groups 
at 2, 4 and 24h after Small incision lenticule extraction. All groups reported a gradual decrease over time. Notably, patients in Group C reported much lower incidence at 2 and $4 \mathrm{~h}$ postoperatively, summarized in Table 5 . At $2 \mathrm{~h}$, the incidence of subjective symptoms in group C was $38.33 \%$ (46 eyes), compared to $66.67 \%$ (80 eyes) in group B and $98.33 \%$ (118 eyes) in group A. At $4 \mathrm{~h}$, the incidence of subjective symptoms in group C was $18.33 \%$ (22 eyes) compared to $35 \%$ (42 eyes) in group B and $68.33 \%$ (82 eyes) in group A.There was no difference between groups after $24 \mathrm{~h}$.

\section{Discussion}

Small incision lenticule extraction is increasingly becoming a popular procedure for the treatment of myopia and myopia astigmatism. Its main advantage is an improved safety profile that lacks corneal flap complications ${ }^{8-10}$. However, it still requires a longer period of time for corneal wound and visual recovery compared to LASIK ${ }^{4,5}$. Currently, little is known about possible influence factors and clinical outcomes in the very early stage of recovery.

In the past few years, it has been reported that treatment using topical $0.1 \%$ fluorometholone resulted in faster improvement of subjective symptom safer corneal surgery ${ }^{11}$ and could reduce the very early inflammatory and wound healing responses post Small incision lenticule extraction ${ }^{12,13}$ via improving the tear film stability and corneal epithelium damage due to decreased corneal sensation. It is reported that the operation mechanical stimulus and laser effect could induce the inflammatory response including the secretion of inflammatory cytokines and corneal edema in the very early postoperative period delaying the corneal wound healing and optical quality recovery ${ }^{14}$. Using $0.1 \%$ fluorometholone and increasing its frequency in the very early stage (within $2 \mathrm{~h}$ postoperatively) could inhibit pro-inflammatory responses and reduce the subsequent corneal edema and opacity.

This study aimed to compare the impact of different different defined daily dose systems of $0.1 \%$ fluorometholone in the very early phase post Small incision lenticule extraction(within $2 \mathrm{~h}$ ) with a follow-up time of $24 \mathrm{~h}$. Currently fluorometholone is not routinely used during the first post-operative day. It is commonly administered four times a day from the second post-operative day onwards ${ }^{4}$ and has shown slow vision 
recovery ${ }^{4,5}$. This study provides valuable information for comparing clinical outcomes such as visual acuity, optical quality and the incidence of subject symptoms. To our knowledge, this is the first report focusing on the acceleration of the recovery process in the very early recovery phase.

A total of 180 patients (360 eyes) undergoing Small incision lenticule extraction were enrolled in our study. They were divided into three groups depending on the frequency of $0.1 \%$ fluorometholone in the first $2 \mathrm{~h}$ after surgery. Every clinical outcome was measured three times at each time point, and got the average value was calculated. Even though all the patients had satisfactory outcomes at $24 \mathrm{~h}$ after surgery, the visual acuity $(\log M A R)$, incidence of subject symptoms and optical quality were significantly different at 2 and $4 \mathrm{~h}$ after surgery. In this study, visual acuity was normalized much faster in group $C$ which was given $0.1 \%$ fluorometholone (q $1 / 2 h$ ). It should be noted that at 2, $4 \mathrm{~h}$ after surgery, the visual acuity of group $\mathrm{C}$ was significantly better compared with the other two groups. Number of eyes recovering higher than $1.0(\log$ MAR 0.0$)$ were also markedly higher in group $\mathrm{C}$ compared to two other groups.

Significant differences in modulation transfer function cutoff, Strehl ratio and objective scattering index were seen between different patient groups at 2 and $4 \mathrm{~h}$ after surgery, which were diminished $24 \mathrm{~h}$ after surgery. We also investigated the incidence of subjective symptoms among the three groups at $2 \mathrm{~h}, 4$ and $24 \mathrm{htime}$ points. The incidence of subjective symptoms, including foreign body sensation, eyes oreness, eye dryness and blurred vision, gradually decreased after surgery in all patient groups. However, the incidence inpatient group $\mathrm{C}$ was significantly lower than the other two groups at 2 and $4 \mathrm{~h}$ post-surgery. A reasonable explanation would be that the increased frequency of fluorometholone use reduced patient discomfort because of reduced inflammation. After $24 \mathrm{~h}$, there was no significant difference between these three groups. Overall, our data showed improved impact of $0.1 \%$ fluorometholone on clinical outcomes and future studies are needed in order to investigate the mechanistic aspect of these events.

\section{Conclusion}


This is the first report examining the clinical outcomes after treatment with $0.1 \%$ fluorometholone in thevery early phase post Small incision lenticule extraction. Our study supports the use of $0.1 \%$ fluorometholone and increasing its frequencyin the very early stage (within $2 \mathrm{~h}$ after Small incision lenticule extraction). Treatment regimen of $0.1 \%$ fluorometholone every half an hour resulted in acceleration of the recovery process, measured by evaluation of visual acuity, modulation transfer function cutoff, Strehl ratio and objective scattering index and patient subjective symptoms. In addition, corneal wound healing, visual and optical quality outcomes were also improved. Overall, our findings can contribute to improved patient satisfaction after surgery and therefore promote the application of Small incision lenticule extraction procedure.

\section{Value Statement}

WHAT WAS KNOWN:

1. Small incision lenticule extraction (Small incision lenticule extraction) procedure has become a popular techniquefor treating myopia and myopia astigmatism.

2. A longer period of time is required for visual recovery from Small incision lenticule extraction operation, limiting its wide spread application. WHAT THIS PAPER ADDS:

1. This is the first report examining the clinical outcomes after treatment with $0.1 \%$ fluorometholone in the very early phase after Small incision lenticule extraction procedure.

2. Visual and optical recovery of patients that were treated with $0.1 \%$ fluorometholone $(\mathrm{q} 1 / 2 \mathrm{~h})$ in the first $2 \mathrm{~h}$ after Small incision lenticule extraction were significantly improved in the very early postoperative phase.

\section{$231 \quad$ Funding}

232 The authors did not receive any financial support from any public or private sources.

\section{Conflicts of interest}

234 The authors declare that they have no competing interests.

Ethics approval and consent to participate

236 All interventions approved by the Institutional Review Board of Fujian Medical 237 University (Fuzhou, China), and were conducted according to the principles in the 238 Declaration of Helsinki. 
240 Informed consent was obtained from all individual participants included in the study.

241 Availability of data and material

242 All data are available and stored by the authors. If someone wants to request the data

243 from this study, please contacte with Zhengwei Shen (E-mail:

244 linqh19870624@163.com)

245 Consent for publication

246 Written consent was obtained from the patient for publication of this manuscript and 247 any accompanying images. A copy of the written consent is available for review by 248 the Editor.

249 Competing interests

250 The authors declare that they have no competing interests.

\section{Acknowledgements}

252 Not applicable.

\section{Contributions}

254 A. B. and C.D. wrote the main manuscript text and E.F. prepared figures 1. All 255 authors reviewed the manuscript.

\section{References}

1. Shen Z,Shi K, Yu Y, et al. Small Incision Lenticule Extraction Versus Fem to Second Laser-assisted in Situ Keratomileusis (FS-LASIK) for Myopia: A System Aticreview and Meta-analysis. PLoS One2016;11:e158176

2. Dong Z, Zhou X, Wu J, et al. Small Incision Lenticule Extraction (SMILE) and Fem to Second Laser Lasik: Comparison of Corneal Wound Healing and Inflammation. Br J Ophthalmol 2014; 98:263-69.

3. Yan H, Gong LY, Huang W, Peng YL. Clinical Outcomes of Small Incision Lenticule Extraction Versus Fem to Second Laser-assisted Lasik for Myopia: A Meta-analysis. Int J Ophthalmol 2017;10:1436-45.

4. Kamiya K, Shimizu K, Igarashi A, Kobashi H. Visual and Refractive Outcomes of Fem to second Lenticule Extraction and Small-incision Lenticule Extraction for 
Myopia. Am J Ophthalmol2014;157:128-34 e2

5. Liu T, Lu G, Chen K, et al. Visual and Optical Quality Outcomes of Smile and FS-LASIK for Myopia in the Very Early Phase After Surgery. BMC Ophthalmology 2019;19:88

6. Mifflin MD, Betts BS, Frederick PA, et al. Efficacy and Safety of A 3-month Loteprednol Etabonate $0.5 \%$ Gel Taper for Routine Prophylaxis After Photorefractive Keratectomy Compared to A 3-month Prednisolone Acetate 1\% and Fluorometholone 0.1\% Taper. Clin Ophthalmol 2017;11:1113-18.

7. Qin Q, Bao L, Yang L, et al. Comparison of Visual Quality After Evo-icl implantation and Smile to Select the Appropriate Surgical Method for High Myopia. BMC Ophthalmology 2019;19:21

8. Li M, Li M, Chen Y, et al. Five-year Results of Small Incision Lenticule Extraction (smile) and Fem to Second Laser Lasik (FS-Lasik) for Myopia. Acta Ophthalmol 2019;97:e373-80

9. Han T, Xu Y, Han X, et al. Three-year Outcomes of Small Incision Lenticule Extraction (smile) and Fem to Second Laser-assisted Laser in Situ Keratomileusis (FS-Lasik) for Myopia and Myopic Astigmatism. $\mathrm{Br} \mathrm{J}$ Ophthalmol 2019;103:565-8

10. Liu M, Chen Y, Wang D, et al. Clinical Outcomes after Smile and Fem to Second Laser-assisted Lasik for Myopia and Myopic Astigmatism: Aprospective Randomized Comparative Study. Cornea 2016;35:210-6

11. Lin T, Gong L. Topical Fluorometholone Treatment for Ocular Dryness in Patients with Sjögren Syndrome: A Randomized Clinical Trial in China. Medicine (Baltimore) 2015;94:e551

12. Luft N, Schumann RG, Dirisamer M, et al. Wound Healing, Inflammation, and Corneal Ultrastructure after Smile and Fem to Second Laser-assisted Lasik: Ahuman Ex Vivo Study. J Refract Surg 2018;34:393-9

13. Liu YC, Teo EP, Lwin NC, et al. Early Corneal Wound Healing and Inflammatory Responses After Smile: Comparison of the Effects of Different Refractive Corrections and Surgical Experiences. J Refract Surg 2016;32:346-53 
299 14. Wei S, Wang $\mathrm{Y}, \mathrm{Wu} \mathrm{D}$, et al. Ultrastructural Changes and Corneal Wound $300 \quad$ Healing After Smile and Prk Procedures. Curr Eye Res2016;41:1316-25

\section{Figure Legends:}

304 Fig 1.Number of eyes recovering higher than 1.0 (logMAR 0.00) among different 305 patient groups. 
Figures

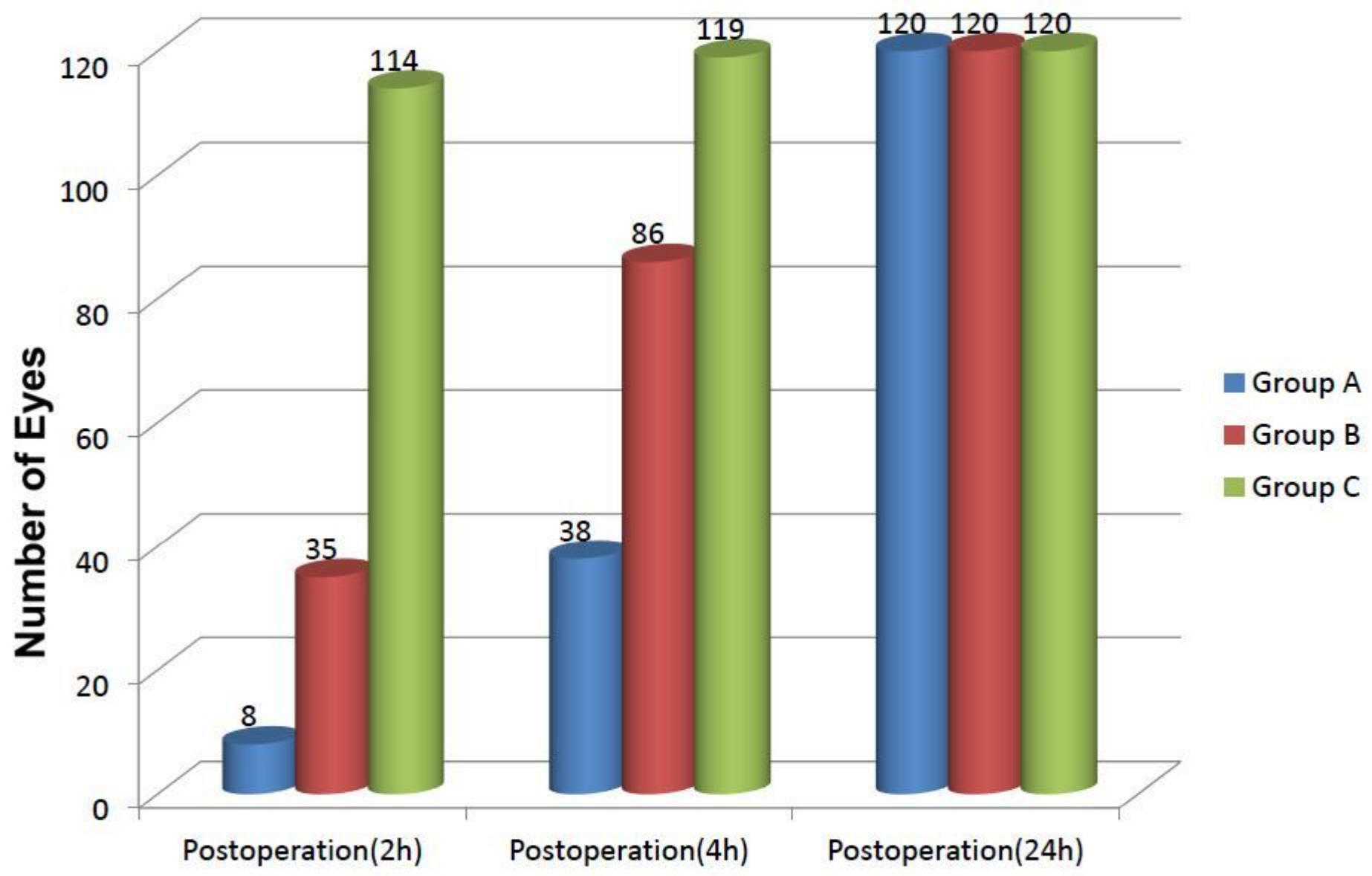

Figure 1

Number of eyes recovering higher than 1.0 (logMAR 0.00) among different patient groups. 